\title{
Soccer Syndrome - 2: Common Innominate Malalignments and Its Manual Diagnostic Techniques in Pelvic Malalignments Syndrome
}

\author{
Ganesh Elumalai $^{1,}$,, Maria Declaro ${ }^{1}$, Sanjoy Sanyal ${ }^{2}$, Melchor Bench Bareng ${ }^{3}$, \\ Aminuddin Mohammad ${ }^{4}$ \\ ${ }^{1}$ Department of Anatomy, College of Medicine, Texila American University, Georgetown, South America \\ ${ }^{2}$ Department of Neuroscience, College of Medicine, Texila American University, Georgetown, South America \\ ${ }^{3}$ Department of Biology, College of Medicine, Texila American University, Georgetown, South America \\ ${ }^{4}$ Department of Biochemistry, College of Medicine, Texila American University, Georgetown, South America
}

Email address:

physiovesalius@gmail.com (G. Elumalai)

\section{To cite this article:}

Ganesh Elumalai, Maria Declaro, Sanjoy Sanyal, Melchor Bench Bareng, Aminuddin Mohammad. Soccer Syndrome - 2: Common Innominate Malalignments and Its Manual Diagnostic Techniques in Pelvic Malalignments Syndrome. American Journal of Sports Science. Vol. 3, No. 6, 2015, pp. 120-127. doi: 10.11648/j.ajss.20150306.14

\begin{abstract}
The innominate malalignments are most common in sports injuries and even in every client who presents with low back \& pelvis pain or dysfunction. In this study, we used simple bony palpation method to assess the various innominate malalignments in football players, which was fails to observe in previous work of various authors. While there are many methods to determine pelvic symmetry or asymmetry, this method is very simple one and that consistently works well for me. This study includes the healthy football players from National Football Club (NFC) of our institution, who were training or playing during the period of investigation (September 2014 - May 2015) $(n=40)$ were eligible for participation. At entry to the study, this eligible sample had a mean age of $22.2 \pm 3.9$ years, height of $175.8 \pm 6.6 \mathrm{~cm}$, and mass of $87.5 \pm 7.1 \mathrm{~kg}$. The number of eligible participants at each time point was 40 for all the four session time, point 1 (T1; start of preseason-3) and point 2 (T2; end of season-3). Subjects were limited to men who had a normal muscle strength and Range of Motion (ROM) of the back and lower extremities and who had no history of orthopaedic or neurologic disorders. All the individuals are subject to gain access the malalignment of both Innominate bones of the bony pelvis unit. In addition, to justify our diagnosis we access the muscles of the functional slings and the muscles around the bony pelvis. The techniques were repeated in different position and also performed for two times per day and continued for fifteen days to standardize. In this study, we observed, $92.5 \%$ i.e., the majority of the soccer's are suffering with multiple innominate malalignments. The soccer's suffering with single component malalignment account for about $25 \%$ includes the isolated Rotational, Upslip or Tilt innominate stucks and soccer's who had the normal innominate at the end of the season is only about $7.5 \%$. Conclusion: This method of assessing the pelvic malalignments until then not documented. In this sense, the objective of this study is to analyze and document the different presentations in pelvic malalignments which are common in soccer players and its simple method of evaluation is the literature state of the art. The present study may provide useful information to analyze common presentations of pelvic malalignments in different sports.
\end{abstract}

Keywords: Pelvic Malalignment, Anterior Pelvic-Tilt, Posterior Pelvic-Tilt, Innominate Upslip

\section{Introduction}

When it comes to idiopathic pain in the low back, we all see essentially the same thing. In 1982 White suggested "It may well be that idiopathic backache will be found to be caused by some condition that is a subtle variation from normal. Otherwise, we probably would have found the cause already. If back pain were caused by a highly unusual condition, then fewer people would suffer from this disorder." 
The lower back is an incredibly complex area, which is why so many people suffer from low back pain. Statistics show that it's the chief complaint of over half of our patients. Lower back problems require a comprehensive approach, looking at muscle tone and function; joint integrity (of at least the lower back and lower extremity); referred pain patterns; and biopsychosocial issues (Marc Heller, DC).

The pelvic malalignment syndrome is the most common in sports injuries and even in every client who presents with low back \& pelvis pain or dysfunction. According to Kristine Boyle Kristine Boyle -Walker, palpation and Mobility tests have shown poor reliability and validity. But, in our previous study, we used simple bony palpation method to assess the various pelvic malalignments in football players and it is consistently works well for me and other manual practitioners. The present study indicates there are various other representations in the innominate bone malalignments in soccer players.

What musculoskeletal issue could result in chronic low back pain, chronic muscle strains, lower extremity tendinopathies, periscapular pain and tightness, glenohumeral and shoulder girdle pain, or tension headaches? I am sure you can think of a few possibilities, but few can result in all. Often, when a patient reports to our care with one of the aforementioned we immediately think locally. Unfortunately, the real problem could be pelvic upslip, anterior pelvic innominate, or both. Despite being oft-overlooked, these malalignments are not hard to identify if you know what to look for (stone athletic medicine: 2014/05).

While there are many methods to determine pelvic symmetry or asymmetry, this method is very simple one and that consistently works well for me. Our method of assessing the pelvic malalignments, even works well to the common individuals suffering with low back \& pelvis pain or dysfunction due to various pelvic malalignment presentation.

Pelvic tilt is often quantified using the angle between the horizontal and a line connecting the anterior superior iliac spine (ASIS) and the posterior superior iliac spine (PSIS). Although this angle is determined by the balance of muscular and ligamentous forces acting between the pelvis and adjacent segments, it could also be influenced by variations in pelvic morphology. The primary objective of this study was to establish how such variation may affect the ASIS-PSIS measure of pelvic tilt. In addition, we also investigated how variability in pelvic landmarks may influence measures of innominate asymmetries.

A standard method of assessing the innominate malalignments is with a horizontal line drawn from the anterior superior iliac spine (ASIS) to the posterior superior iliac spine (PSIS). Although this angle is dependent on the muscular and ligamentous forces that act between the pelvis and adjacent segments, it is also dependent on the relative position of the two bony landmarks (ASIS and PSIS) on the innominate bone.
'Pelvic tilt is often quantified using the angle between the horizontal and a line connecting the anterior superior iliac spine (ASIS) and the posterior superior iliac spine (PSIS). Although this angle is determined by the balance of muscular and ligamentous forces acting between the pelvis and adjacent segments, it could also be influenced by variations in pelvic morphology'. The normal innominate bone is tilted anteriorly with $4^{0}-7^{0}$ in male and $7^{0}-10^{0}$ in female as shown in the Figure-1 (Bradeley 2015).

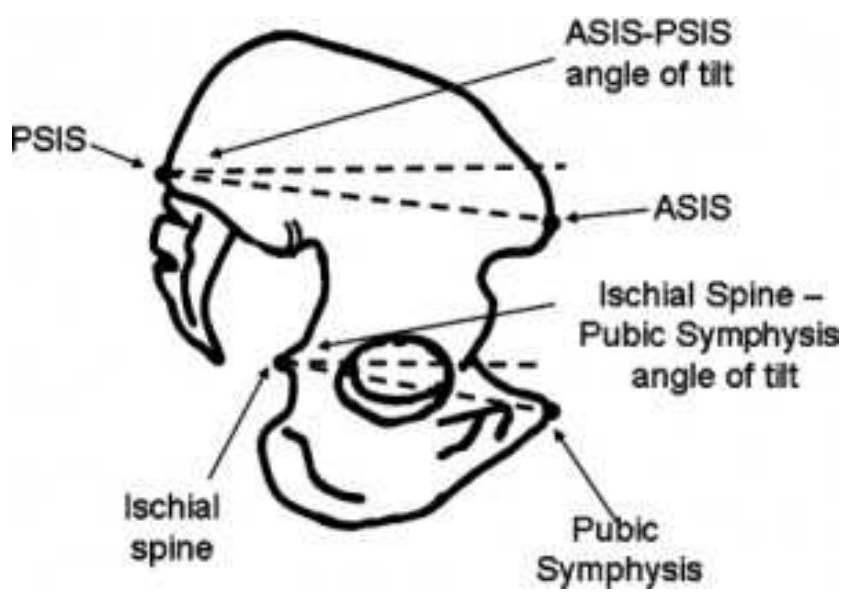

Figure 1. Normal position of Innominate bone.

\section{Materials \& Methods}

The healthy football players from National Football Club (NFC) of our institution, who were undergoing training or playing during the period of investigation (September 2014 - May 2015) $(\mathrm{n}=40)$ were eligible for participation in the study. At entry to the study, this eligible sample had a mean age of $22.2 \pm 3.9$ years, height of $175.8 \pm 6.6 \mathrm{~cm}$, and mass of $87.5 \pm 7.1 \mathrm{~kg}$. The number of eligible participants at each time point was 40 for all the two session time, period - 1 (T1; Start of Preseason - 3) and period - 2 (T2; End of Season - 3). Subjects were limited to men who had the normal muscle strength and normal ROM of the back and lower extremities and who had no history of orthopaedic or neurological disorders. All subjects were instructed to restrict excessive physical activity on the day of testing, such as recreational running and bicycling, and to wear gym trunks for the tests.

On the day of testing, all subjects reviewed and signed informed consent forms for the study. On assessment we taught the subjects how to perform the movements to gain access to the malalignment of both Innominate bones of the bony pelvis unit. In addition, to justify our diagnosis we access the muscles of the functional slings and the muscles around the bony pelvis related to the malalignment of the pelvic unit (Abdolhamid Daneshjoo, 2013), (Julie Hides, 2012), (Fernando Idoate, 2011). We encouraged all the subjects to relax and told them that the movements would not be judged as "good" or "bad." We believed these verbal cues decreased subjects' anxiety and, therefore, helped to standardize the testing. The techniques were repeated in 
different positions and also performed two times per day and continued for fifteen days to standardize.

\section{Muscles Responsible to Maintain the Symmetry of Innominate Bone}

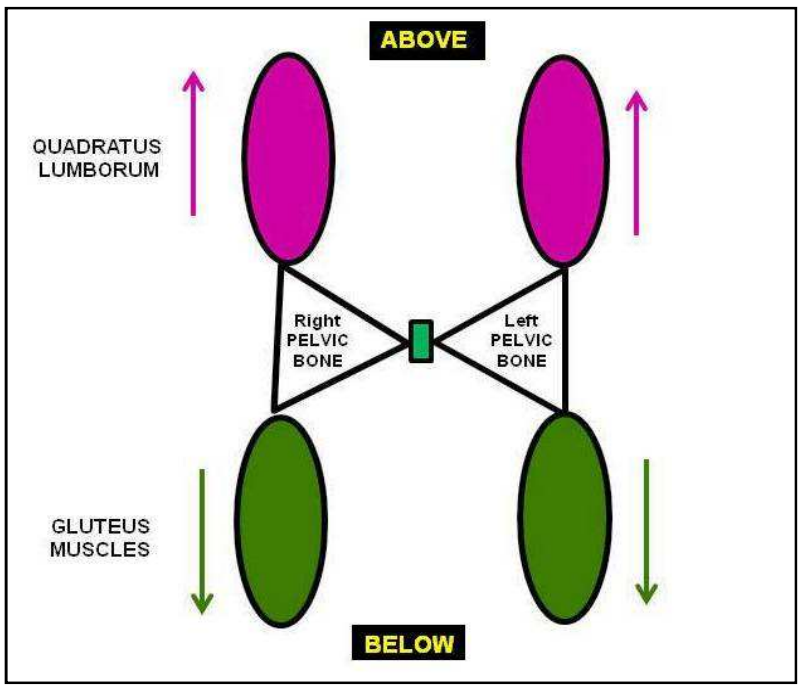

Figure 2. Muscles responsible for symmetry of Pelvic bone.

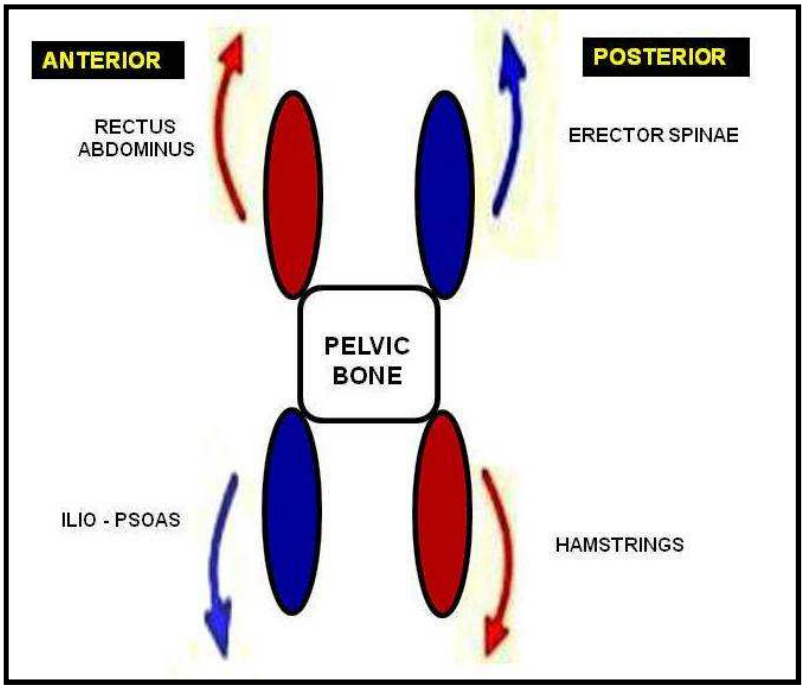

Figure 3. Muscles responsible for lateral stability of Pelvic bone.

\section{Our Previous Observations of Innominate Bone Malalignments}

The previous observations on the innominate malalignments are: (in relation to the Right innominate) 1. Low right ASIS, high right PSIS, near level Intercristal line:

Anterior rotation of the right innominate or posterior rotation of the left innominate

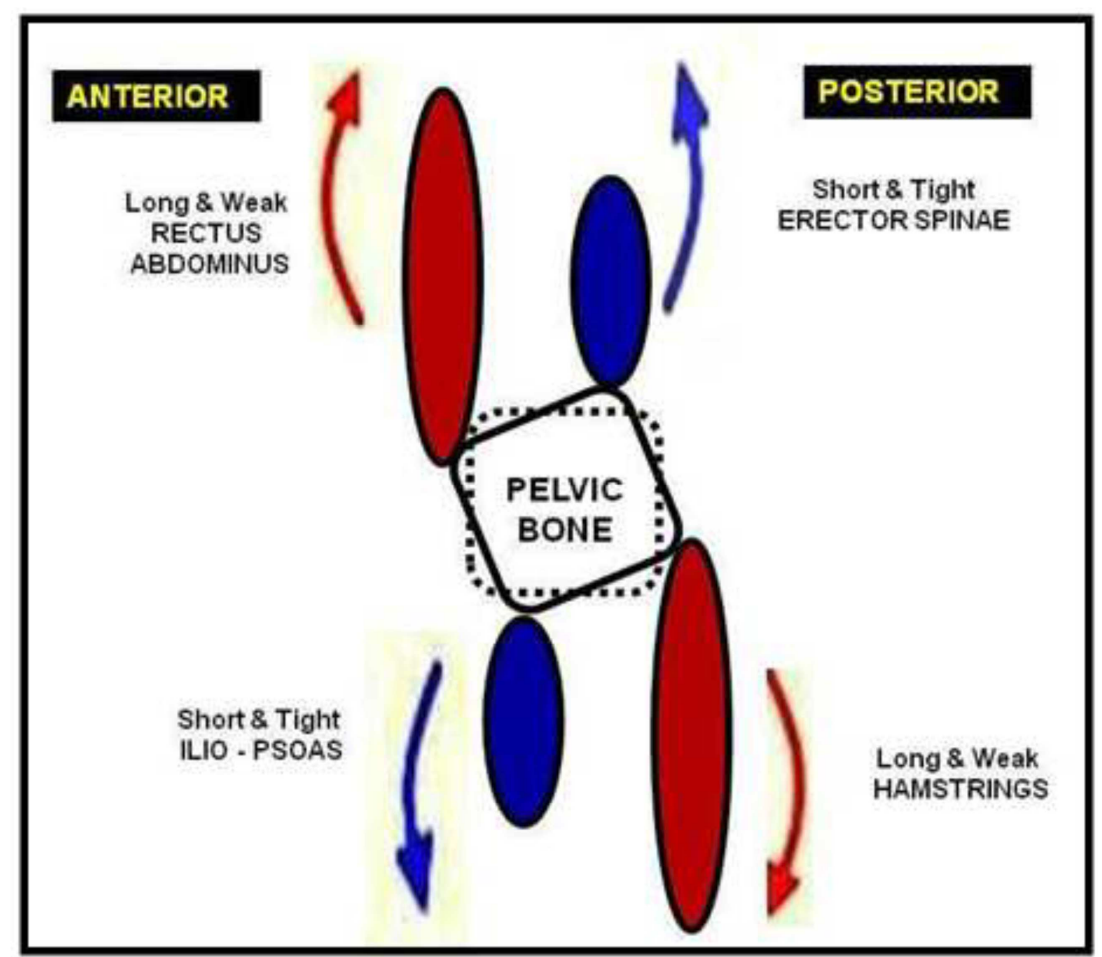

Figure 4. Muscles involved in Anterior Innominate stuck.

2. High right ASIS, low right PSIS, near level Intercristal line:

Posterior rotation of the right innominate or anterior rotation of the left innominate 


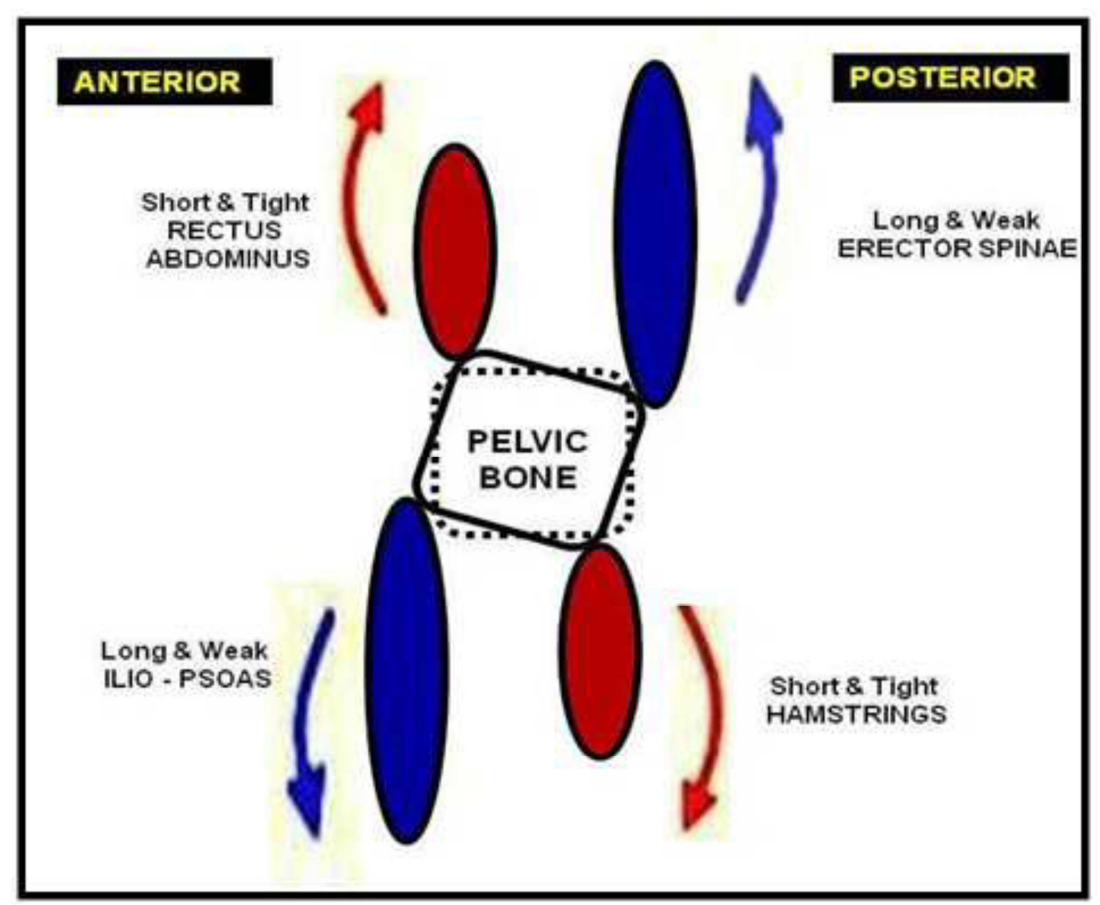

Figure 5. Muscles involved in Posterior Innominate stuck.

3. High right ASIS \& PSIS, with right superior pubic:

Right innominate up-slip with supra-pubic dysfunction

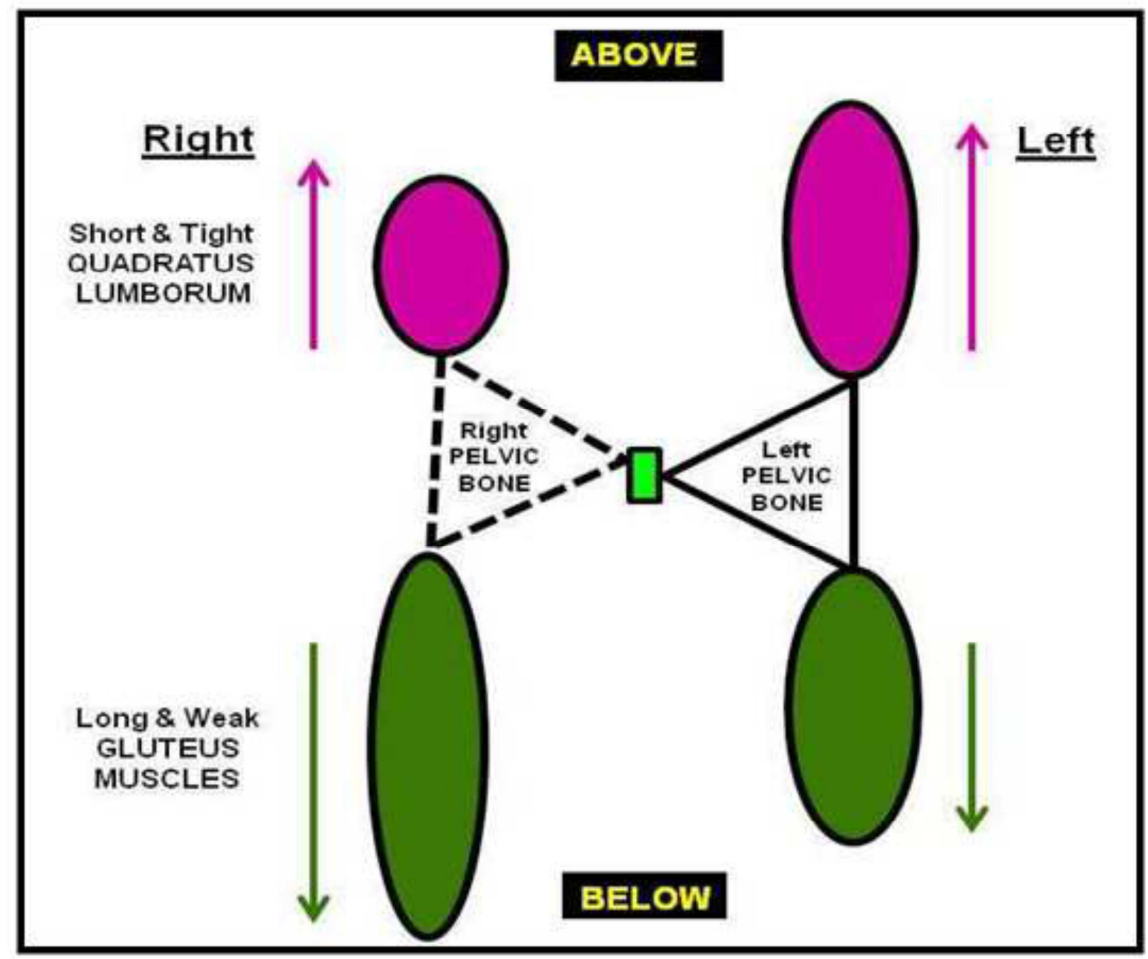

Figure 6. Muscles involved in Right Innominate Upslip stuck.

4. Level ASIS's with high right PSIS and high right iliac crest:

Right innominate anterior rotation with a right up-slip 


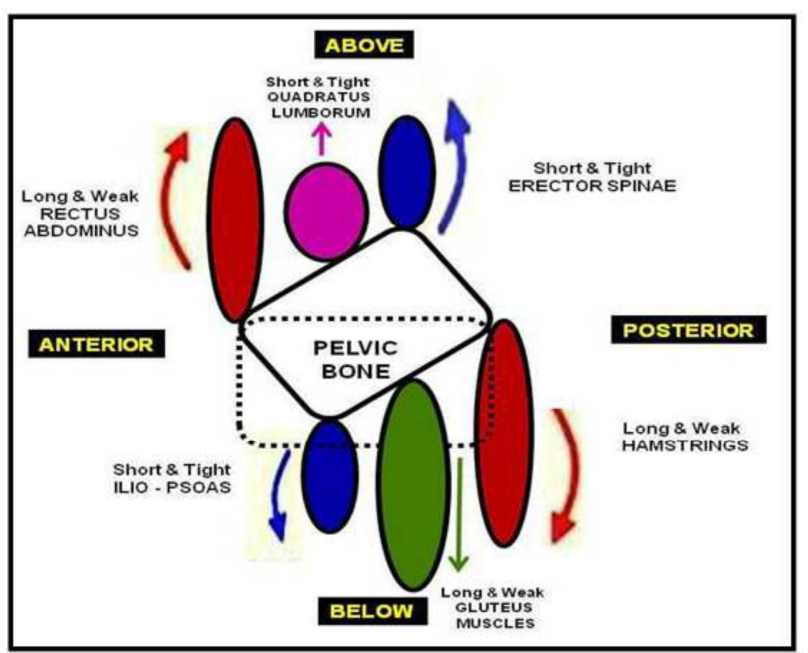

Figure 7. Muscles involved in Right Innominate Upslip \& Anterior rotational stuck.

5. High right ASIS with level PSIS's and high right crest: Right innominate posterior rotation with up-slip

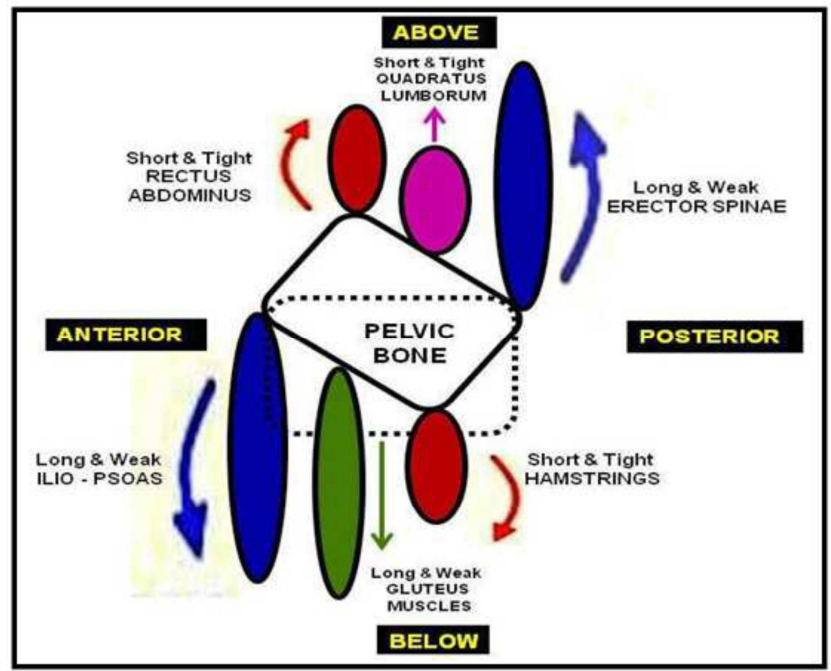

Figure 8. Muscles involved in Right Innominate Upslip \& Posterior rotational stuck.

6. Medial right ASIS with Lateral right PSIS

Right Inflare innominate

7. Lateral right ASIS with Medial right PSIS

Right Outflare innominate

The above mentioned presentation may appear in isolation or in combination with one or both of the others. The foreseen seven possibilities are applicable even in the left side innominate bone.

\section{Our Current Observations of Innominate Bone Malalignments}

The assessment of innominate bone is followed by the Bony Pelvis Malalignment Assessment Chart of Ganesh et al (2014).

The common presentation may appear in isolation or in combination with one or both of the others. For example, an 'Upslip' appears on its own in about \%, in combination with either 'rotational malalignment' or 'flare' or both in another $10 \%$, for a total of $20 \%$ overall. In this study, the common 'Innominate malalignment' refers to fixation of an innominate bone relative to the sacrum in excessive anterior or posterior rotation in the sagittal plane. Such rotation can affect an innominate on one side only but is more likely to be seen in association with:

1. Compensatory rotation of the contralateral innominate around the coronal axis in the sagittal plane. (e.g. Right side anterior rotation with Left side Posterior rotation)

2. Compensatory rotation of the contralateral innominate around the vertical axis in the transverse plane. (e.g. Right side anterior rotation with outflare \& Left side Posterior rotation with inflare)

3. Upslip of innominate with the displacement of the pubic bone superiorly, relative to each other

4. Upslip with compensatory rotation of the contralateral innominate around the coronal axis in the sagittal plane. (e.g. Upslip \& Right side anterior rotation with Left side Posterior rotation)

5. Upslip with compensatory rotation of the contralateral innominate around the vertical axis in the transverse plane. (e.g. Upslip \& Right side anterior rotation with outflare \& Left side Posterior rotation with inflare)

6. Both the innominate in fixed with Anterior (Anteriortilt), Posterior (Posterior-tilt) and Lateral (Lateral-tilt, without the displacement of the pubic bone superiorly)

\section{Clinical Observations}

1. Isolated Rotational Malalignments in coronal axis \& sagittal plane.

- Low right ASIS, high right PSIS, near level Intercristal line (Right anterior rotational stuck)

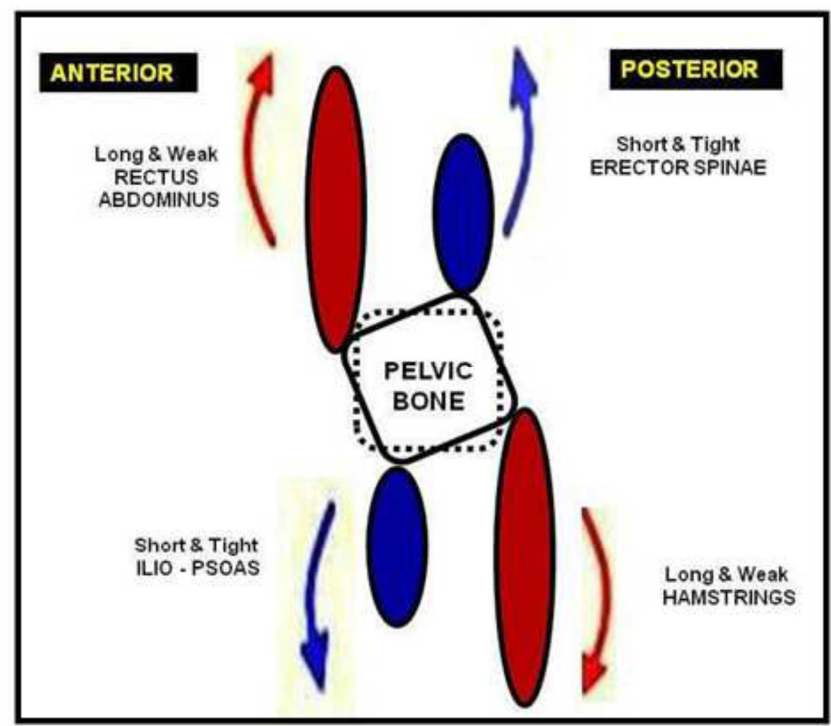

Figure 9. Muscles involved in Right Innominate Isolated Anterior rotational stuck. 
- High right ASIS, low right PSIS, near level Intercristal line (Right Posterior rotational stuck)

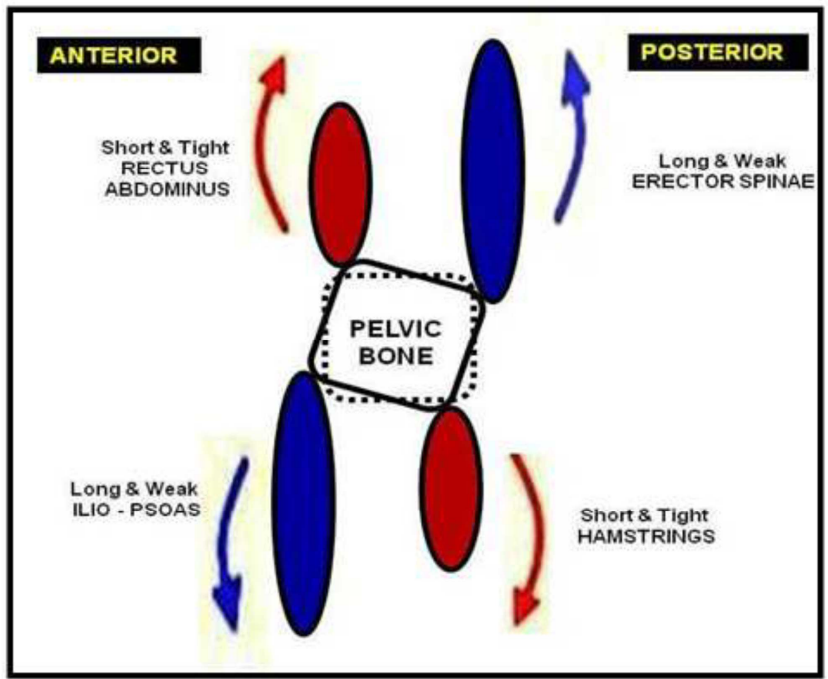

Figure 10. Muscles involved in Right Innominate Isolated Posterior rotational stuck.

2. Combined rotational Malalignments of coronal axis \& sagittal plane with vertical axis \& transverse plane.

- Low \& lateral right ASIS, high \& medial right PSIS, with near level Intercristal line. (Right side anterior rotational stuck with outflare)

- Low \& medial right PSIS, high \& lateral right ASIS, with near level Intercristal line. (Right side Posterior rotational stuck with inflare)

3. Isolated Upslip of innominate with the displacement of the pubic bone superiorly, relative to each other.

- High right ASIS \& PSIS, with right superior pubic: (Right innominate up-slip stuck with supra-pubic dysfunction)

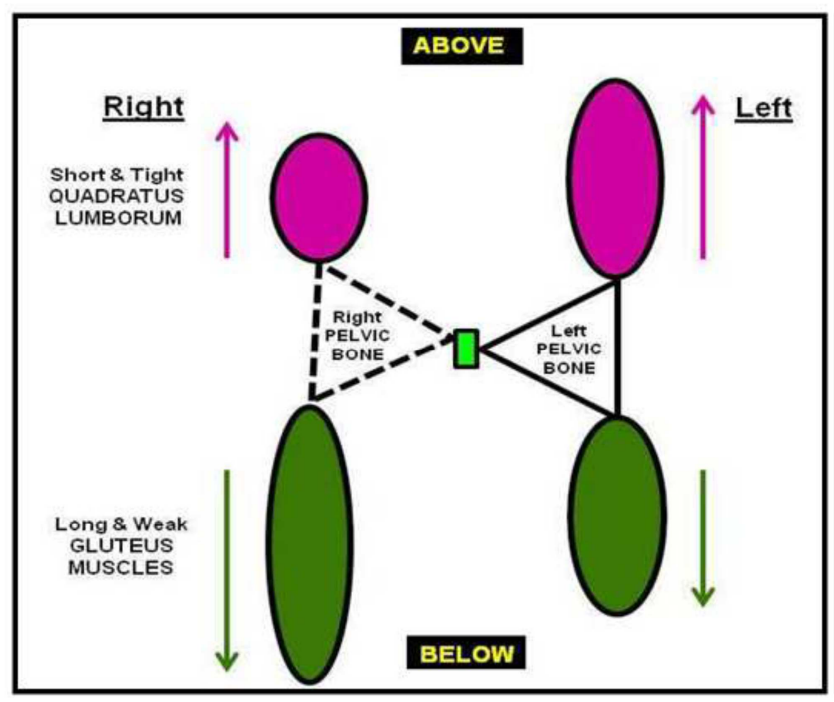

Figure 11. Muscles involved in Right Innominate Isolated Upslip stuck.

4. Combined Upslip with rotational Malalignments in coronal axis \& sagittal plane.
- Right Upslip \& level / low right ASIS, high right PSIS, with right Suprapubic dysfunction. (Right innominate Upslip with Anterior rotational stuck)

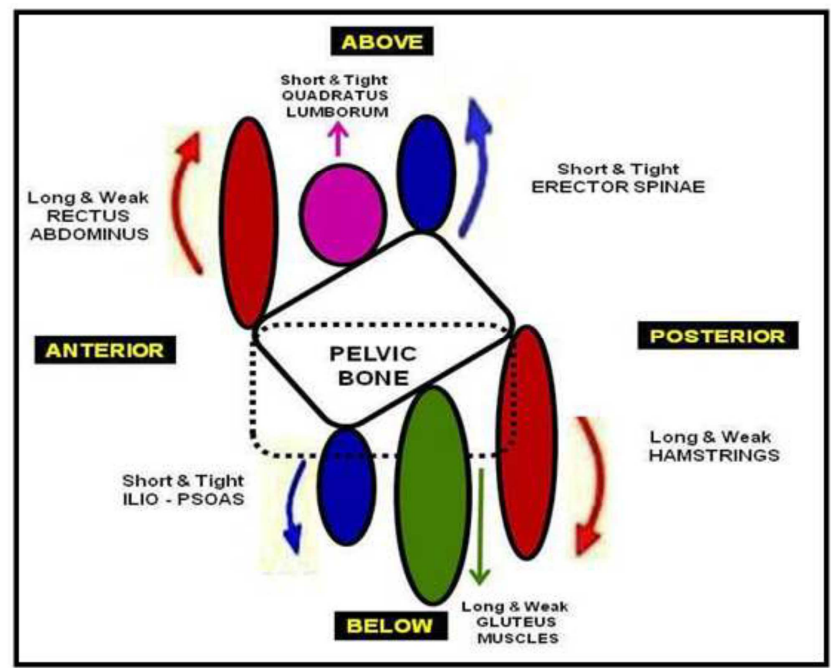

Figure 12. Muscles involved in Right Innominate Upslip with Anterior rotational stuck.

- Right Upslip \& High right ASIS, level / low right PSIS, with right Suprapubic dysfunction. (Right innominate Upslip with Posterior rotational stuck)

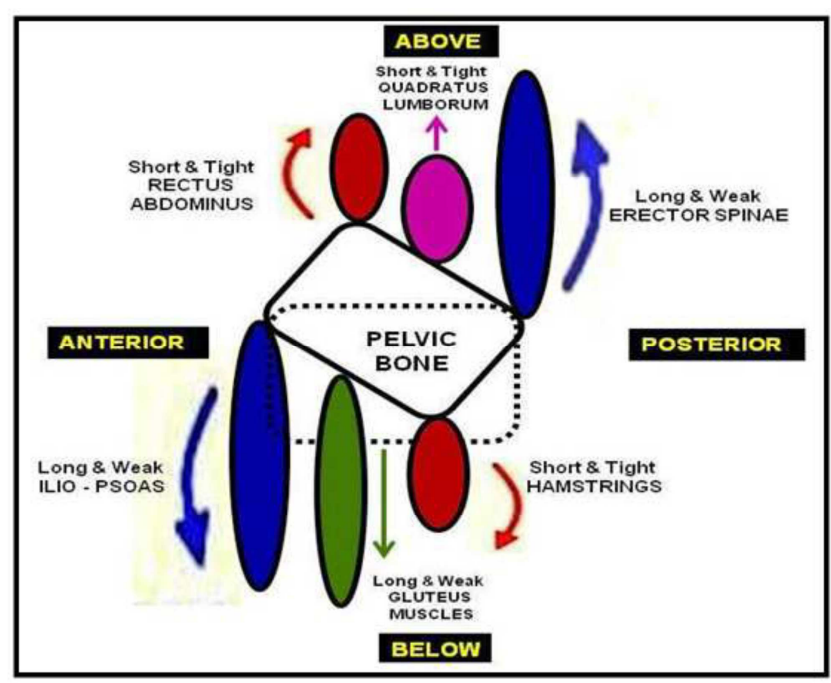

Figure 13. Muscles involved in Right Innominate Upslip with Posterior rotational stuck.

5. Combined Upslip and rotational Malalignments of coronal axis \& sagittal plane with vertical axis \& transverse plane.

- Right Upslip with level / low \& lateral right ASIS, high \& medial right PSIS, with right Suprapubic dysfunction. (Right side innominate Upslip and anterior rotational stuck with outflare)

- Right Upslip with level / low \& medial right PSIS, high \& lateral right ASIS, with right Suprapubic dysfunction. (Right side innominate Upslip and posterior rotational stuck with inflare) 
6. Bilateral Low ASIS \& High PSIS, near level Intercristal line \& Pubic symphysis.

- Anterior tilted pelvis

7. Bilateral Low PSIS \& High ASIS, near level Intercristal line \& Pubic symphysis
- Posterior tilted pelvis

8. Unilateral high ASIS \& PSIS, Ipsilateral high Intercristal line \& Neutral Pubic symphysis.

- Lateral tilted pelvis

\section{Observations}

Table 1. Period - 1 (Start of Preseason-3).

\begin{tabular}{llll}
\hline S.NO & INNOMINATE MALALIGNMENTS & Frequency (n=40) & Percentage (\%) \\
\hline 1 & Isolated Anterior rotational stuck & 1 & 2.5 \\
2 & Isolated Posterior rotational stuck & 0 & 0 \\
3 & Anterior rotational stuck with Outflare & 2 & 5.0 \\
4 & Posterior rotational stuck with Inflare & 1 & 2.5 \\
5 & Isolated Upslip & 0 & 0 \\
6 & Upslip with Anterior rotational stuck & 1 & 2.5 \\
7 & Upslip with Posterior rotational stuck & 0 & 0 \\
8 & Upslip with Anterior rotational stuck with Outflare & 1 & 0 \\
9 & Upslip with Posterior rotational stuck with Inflare & 0 & 2.5 \\
10 & Anterior tilted pelvis & 1 & 0 \\
11 & Posterior tilted pelvis & 0 & 0 \\
12 & Lateral tilted pelvis & 0 & 82.5 \\
13 & Neutral pelvis & 33 & $100 \%$ \\
\hline
\end{tabular}

Table 2. Period - 2 (End of Season-3).

\begin{tabular}{llll}
\hline S.NO & Innominate Malalignments & Frequency (n=40) & Percentage (\%) \\
\hline 1 & Isolated Anterior rotational stuck & 3 & 7.5 \\
2 & Isolated Posterior rotational stuck & 1 & 2.5 \\
3 & Anterior rotational stuck with Outflare & 11 & 27.5 \\
4 & Posterior rotational stuck with Inflare & 5 & 12.5 \\
5 & Isolated Upslip & 1 & 2.5 \\
6 & Upslip with Anterior rotational stuck & 3 & 7.5 \\
7 & Upslip with Posterior rotational stuck & 1 & 2.5 \\
8 & Upslip with Anterior rotational stuck with Outflare & 5 & 12.5 \\
9 & Upslip with Posterior rotational stuck with Inflare & 2 & 5.0 \\
10 & Anterior tilted pelvis & 3 & 7.5 \\
11 & Posterior tilted pelvis & 1 & 2.5 \\
12 & Lateral tilted pelvis & 1 & 2.5 \\
13 & Neutral pelvis & 3 & 7.5 \\
Total & & 40 & $100 \%$ \\
\hline
\end{tabular}

\section{Discussion}

The Innominate malalignments are most common in soccer players. An understanding of this 'malalignment syndrome' requires knowledge of the common presentations of malalignments and the techniques used to diagnose and treat.

According to the e-source on "Pelvic evaluation 2010", there are four common presentations on innominate malalignment. The previous observation of this study noticed that there are seven possible innominate malalignment presentations. But, the current study observed that, there are thirteen (including normal pattern) different alignments in innominate bone of soccer players.

The present identified that there is five different observations in the Isolateral and combinational Upslip stucks and innominate stucks in Anterior, Posterior and Lateral tilts, which was failed to notice in Abdolhamid Daneshjoo, 2013; Fernando Idoate, 2011; Julie Hides, 2012; Ganesh,2014.
The present study shows that Anterior and Posterior Innominate stuck can occur due to the changes in the strength of Quadriceps and Hamstring muscles which is similar to the previous work done by Abdolhamid, 2013. According to Abdolhamid, 2013, the physical performance and movement patterns of Soccer players may change the balance of strength in both the legs, not particularly to the dominant kicking leg which is contradict to the current study in which we identified the muscle imbalance and malalignment is more common in preferred kicking leg. Rectus abdominis undergoes hypertrophy in the soccer players which is a common observation between the current study and Fernando, 2011.In the present study we observed that change in the strength and bulk of Rectus abdominis in the preferred kicking leg results in posterior innominate stuck in the soccer players but Fernando, 2011 stated that changes in the strength and bulk of rectus abdominis is bilateral and asymmetrical.

The above mentioned facts, observations and results indicated that an imbalance in the key muscles around the bony pelvis will affect the innominate stability. 


\section{Conclusion}

Pelvic tilt is often quantified using the angle between the horizontal and a line connecting the anterior superior iliac spine (ASIS) and the posterior superior iliac spine (PSIS). Although this angle is determined by the balance of muscular and ligamentous forces acting between the pelvis and adjacent segments, it could also be influenced by variations in pelvic morphology. The primary objective of this study was to establish how such variation may affect the ASISPSIS measure of pelvic tilt. In addition, we also investigated how variability in pelvic landmarks may influence measures of innominate asymmetries.

The present study is mainly focused on the common presentations and manual diagnostic techniques for innominate malalignments. We used simple bony palpation method to assess the various innominate malalignments in football players. While there are many methods to determine pelvic symmetry or asymmetry, this method is very simple one and that is consistently works well for me. Our method of assessing the pelvic malalignments, even works well to the common individuals suffering with low back \& pelvis pain or dysfunction due to various pelvic malalignment presentation.

To be masterly skilled in our current techniques of evaluating the pelvic malalignment it is very simple and essential that you will need to be able to locate the anterior superior iliac spines (ASIS) and posterior superior iliac spines (PSIS), able to judge the levelness of both iliac crests when viewing from the front or rear even in different postures like standing, lying in supine \& prone also while performing the movements during the assessment.

The repeated measurement and analysis proves that the "asymmetry of pelvis" which may be "ipsilateral" or "contralateral" to the preferred kicking leg. The results showed an imbalance of the key muscles associated with Pelvic stability. In this study, we observed, $92.5 \%$ i.e., the majority of the soccer's are suffering with multiple innominate malalignments. The soccer's suffering with single component malalignment account for about $25 \%$ includes the isolated Rotational, Upslip or Tilt innominate stucks and soccer's who had the normal innominate at the end of the season is only about $7.5 \%$.

Noted, the pelvic rotations are relative and it is not always necessary to know which side is the dysfunctional side if you are treating them, as you should treat both sides of the pelvis. To justify our diagnosis we access the muscles of the functional slings and the muscles around the bony pelvis related to the malalignment of the pelvic unit. The techniques were repeated in different position and also performed for two times per day and continued for fifteen days to standardize.

However, this method of assessing the pelvic malalignments is recently documented by our previous work (Ganesh, 2014). In this sense, the current study is mainly focused on the analysis and documentation of the different common presentations in innominate malalignments which are common in soccer players and its simple method of evaluation is the literature state of the art. The present study may provide useful information to analyze common presentations of innominate malalignments in different sports.

\section{References}

[1] Abdolhamid Daneshjoo et al., Bilateral and Unilateral Asymmetries of Isokinetic strength and Flexibility in Male Young Professional Soccer Players. Journal of Human Kinetics.36, 45-53. 2013.

[2] Bradeley.http://www.biomechanicsacademy.com/wpcontent/uploads/2015/07/A CRITICAL-REVIEW-OF-THEPREECE-SJ-2008-PAPER.pdf

[3] Fernando Idoate et al., Soccer attenuates the Asymmetry of Rectus abdominis Muscle observed in Non- Athletes. PLOS ONE. 6(4), 1-7. 2011.

[4] E. Ganesh et al., Soccer syndrome - Common presentations and manual diagnostic techniques for pelvic malalignment syndrome. American Journal of Sports Science. Science Publishing group. 2(6): 141-154. 2014.

[5] Julie Hides \& Warren Stanton. Muscle imbalance among Elite Australian Rules Football Players, A longitudinal study of changes in trunk muscle size. Journal of Athletic training.47 (3), $314-319.2012$.

[6] marchellerdc.com/pro_resources/Articles/DC_18_SI1_Shears. pdf.

[7] members.nata.org/virtuallibrary/sacroiliac/pdfs/PowerPoint_P resentations/Ilial_Pathology.pdf

[8] Pelvic Evaluation. www.waltfritz seminars.com/myofascial resource/wp-content/uploads/2010/09.

[9] stoneathleticmedicine.com/2014/05/pelvic-upslip-androtation-evaluation-and-treatment/.

[10] White AA. Introduction. White AA, Gordon SL (eds): American Academy of Orthopaedic Surgeons Symposium on Idiopathic Low Back Pain. St. Louis, MO, CV Mosby Co. 1982, p 2. 